\title{
Elastic Moduli of Carbon Nanohorns
}

\author{
Dinesh Kumar, ${ }^{1}$ Veena Verma, ${ }^{2}$ H. S. Bhatti, ${ }^{1}$ and Keya Dharamvir ${ }^{3}$ \\ ${ }^{1}$ Department of Physics, Punjabi University, Patiala, India \\ ${ }^{2}$ Department of Physics, Government College, Naya Nangal, India \\ ${ }^{3}$ Centre for Advance Study in Physics, Panjab University, Chandigarh, India
}

Correspondence should be addressed to Dinesh Kumar, dineshk_2@yahoo.co.in

Received 26 September 2010; Accepted 2 March 2011

Academic Editor: Wanqin Jin

Copyright (C) 2011 Dinesh Kumar et al. This is an open access article distributed under the Creative Commons Attribution License, which permits unrestricted use, distribution, and reproduction in any medium, provided the original work is properly cited.

Carbon nanotube is a special case of carbon nanohorns or carbon nanocones with zero apex angle. Research into carbon nanohorns started almost at the same time as the discovery of nanotubes in 1991. Most researchers focused on the investigation of nanotubes, and the exploration of nanohorns attracted little attention. To model the carbon nanohorns, we make use of a more reliable secondgeneration reactive empirical bond-order potential by Brenner and coworkers. We investigate the elastic moduli and conclude that these nanohorns are equally strong and require in-depth investigation. The values of Young's and Shear moduli decrease with apex angle.

\section{Introduction}

Since the discovery of carbon nanotubes (CNT) in the early 1990s [1], there has been much research work in this field. In a series of reports, CNTs have been found to possess a low density but a high stiffness and a high strength, along with excellent electronic properties. The combination of structural perfection and these desirable properties makes CNTs a popular material for study, and they are expected to have a wide range of applications in electronics, batteries and supercapacitors, hydrogen storage, reinforcements in composites, nanosensors and probes, and electron emission. Research into nanomaterials has also revealed that carbon is a flexible material that can form a variety of structures. In addition to CNTs and fullerenes, it can exist in the form of carbon onions [2], carbon boxes [3], nanocapsules [4], and carbon nanohorns $(\mathrm{CNHs})$ or nanocones $(\mathrm{CNCs})[5,6]$. $\mathrm{CNTs}$, which have zero apex angles, can be treated as a special case of CNCs.

Research into CNCs started almost at the same time as the discovery of CNTs in 1991. The official report of the discovery of isolated CNCs was made in 1993, when Ge and Sattler [5] from the University of Hawaii reported their observations of carbon cones mixed together with tubules on a flat graphite surface. The products were generated by quenching hot carbon vapor on the substrate. Ge and Sattler also predicted that five apex angles, $19.2^{\circ}, 38.9^{\circ}, 60^{\circ}, 83.6^{\circ}$, and $112.9^{\circ}$, could be used to distinguish CNCs. However, only the angle of $19.2^{\circ}$ was found in the opening angles of their samples. The observed cones were up to $24 \mathrm{~nm}$ in length and $8 \mathrm{~nm}$ in base diameter.

At that time, most researchers focused on the investigation of CNTs, and the exploration of CNCs attracted little attention. The findings of Ge and Sattler were largely forgotten until Krishnan et al. [6] verified the existence of the five types of cones, which they generated by the prolysis of hydrocarbons in a carbon arc. These results indicated that the curvature of carbon structures is nearly always determined in the nucleation stage, and in their experiments they demonstrated that by, adding a pentagon, a CNC with a $19.2^{\circ}$ opening angle could grow into a CNT.

The construction of a single-walled CNC can be carried out using a similar strategy to that used to construct a CNT. First of all, the axial and transverse directions are determined by specifying the translational and chiral vectors on the graphene sheet. The graphene sheet is then rolled in the desired direction, and open edges are joined to get CNC. 
TABLE 1: Parameters for the carbon-carbon pair terms.

\begin{tabular}{lcc}
\hline Parameters & Brenner's (20) & Ours (21) \\
\hline$B_{1}(\mathrm{eV})$ & 12388.79197798 & 12300.79197798 \\
$\beta_{1}\left(\AA^{-1}\right)$ & 4.7204523127 & 4.7204523127 \\
$Q(\AA)$ & 0.3134602960833 & 0.3996602960833 \\
$B_{2}(\mathrm{eV})$ & 17.56740646509 & 17.56740646509 \\
$\beta_{2}\left(\AA^{-1}\right)$ & 1.4332132499 & 1.4332132499 \\
$A(\mathrm{eV})$ & 10953.544162170 & 10900.544162170 \\
$B_{3}(\mathrm{eV})$ & 30.71493208065 & 30.71493208065 \\
$\beta_{3}\left(\AA^{-1}\right)$ & 1.3826912506 & 1.3826912506 \\
$\alpha\left(\AA^{-1}\right)$ & 4.7465390606595 & 4.7465390606595 \\
\hline$R=1.8 \AA, D=0.3 \AA$. & &
\end{tabular}

The aim of this strategy is to form a cone using the same procedure of rolling a sector of graphene sheet around the apex and joining the open sides. In rolling a plain sheet, any cone angle can be produced, but this is not so with graphene sheet in which the lattice points are arranged periodically and the honeycomb texture of the graphene has to be considered. For closing the two open sides, the structure should match at the joint line.

The apex angles of cones that are formed in this manner can also be given by $2 \arcsin (1-n / 6)$ or by $2 \arcsin (1-\theta / 360)$, where $n$ is the number of pentagons in the structure and $\theta$ is the disclination angle in degrees. Taking $n$ from 1 to 5 , the apex angles of closed cones are $19.2^{\circ}, 38.9^{\circ}, 60^{\circ}, 83.6^{\circ}$, and $112.9^{\circ}$. In the case of an open-cone structure, a total of six pentagons result in a tube with zero apex angles.

There are different approaches to study the mechanical properties of CNTs. A good number of works have been devoted to determine the Young's modulus of CNTs, for example, using the empirical MD [7-11], tight binding [1214] or ab initio [15-17], and atomistic-based finite-deformation shell theory for single-walled CNTs [18]. A shell theory for carbon nanotubes is based on the interatomic potential and atomic structure [19]. On the other hand, very little theoretical work has been undertaken on the structure of $\mathrm{CNHs}$, and researchers are still struggling hard to establish universally accepted approaches to determine the structure and growth mechanism of CNHs. This work is an attempt to study structure and elastic moduli of CNHs using second-generation REBO potential [20]. Some of the parameters have been modified by us [21] to account for the bond length and cohesive energy for carbon systems. We report here the elastic moduli of carbon nanohorns.

\section{Model Potential}

2.1. Second-Generation Reactive Empirical Bond-Order Potential. The energy of the system is a sum of the energy on each $\mathrm{C}-\mathrm{C}$ bond composed of a repulsive part and attractive part [20]. In this work, we have used an improved "second-generation" form of potential, and the details of this potential energy expression are given below

$$
\begin{gathered}
V_{R}(r)=f_{c}(\mathrm{r})\left(1+\frac{Q}{r}\right) A e^{-\alpha r}, \\
V_{A}(r)=f_{c}(\mathrm{r}) \sum_{n=1,3} B_{n} e^{-\beta_{n} r} .
\end{gathered}
$$

Expressions (1) and (2) are used for repulsive and attractive pair terms, respectively. The subscript $n$ refers to the sum in (2), and $r$ is the scalar distance between atoms. The screened Coulomb function used for the repulsive pair interaction goes to infinity as interatomic distances approach zero. The function $f_{c}(r)$ limits the range of the covalent interactions and assumes a value of one for $f_{c}(r)$ for nearest neighbors and zero for interatomic distances more than 2.1, and value around $R$ is given as

$$
f_{c}(r)= \begin{cases}1, & r<(R-D), \\ \frac{1}{2}-\frac{1}{2} \sin \left[\frac{1}{2} \frac{\pi(r-R)}{D}\right], & (R-D)<r<(R+D), \\ 0, & r>(R+D) .\end{cases}
$$

To reproduce the bond length and cohesive energy, we modify some of the parameters of second-generation potential by Brenner and coworkers. The original parameters yield to the minima at $1.32 \AA$ for carbon-carbon bond, but modified parameters give the minima at $1.42 \AA$. These modified parameters are used to study the carbon nanostructures (see Table 1).

\section{Computational Method}

The CNT can be seen as a special case of a $\mathrm{CNH}$ with zero conical angle. The configurations of several single-walled CNHs and CNT with the same height and top radius but different bottom radii or, alternatively, different conical angles are represented in Figure 1. The six computational cases demonstrate the gradual changes in the apex angle from $112.9^{\circ}$ to $0^{\circ}$, which corresponds to the disclination angle from $60^{\circ}$ to $360^{\circ}$ on the graphene sheet. The dimensions of the $\mathrm{CNHs}$ are carefully chosen to allow comparison with the $(10,10) \mathrm{CNT}(L / D=9.0)[21]$.

The coordinates of carbon atoms are generated for the carbon horns, and using the potential by Brenner and coworkers, the energy of the system is calculated. The system is then relaxed to get a minimum energy configuration. For obtaining elastic moduli, we apply stress or twist to the system and get the second derivative of energy, which in turn is used to calculate the moduli as described in next section. The nanohorns have a variable radius along the height so the stress distribution in them is not uniform but varies according to height.

When the $\mathrm{CNH}$ is in equilibrium, the top level should have the largest stress and the bottom level the smallest, and 


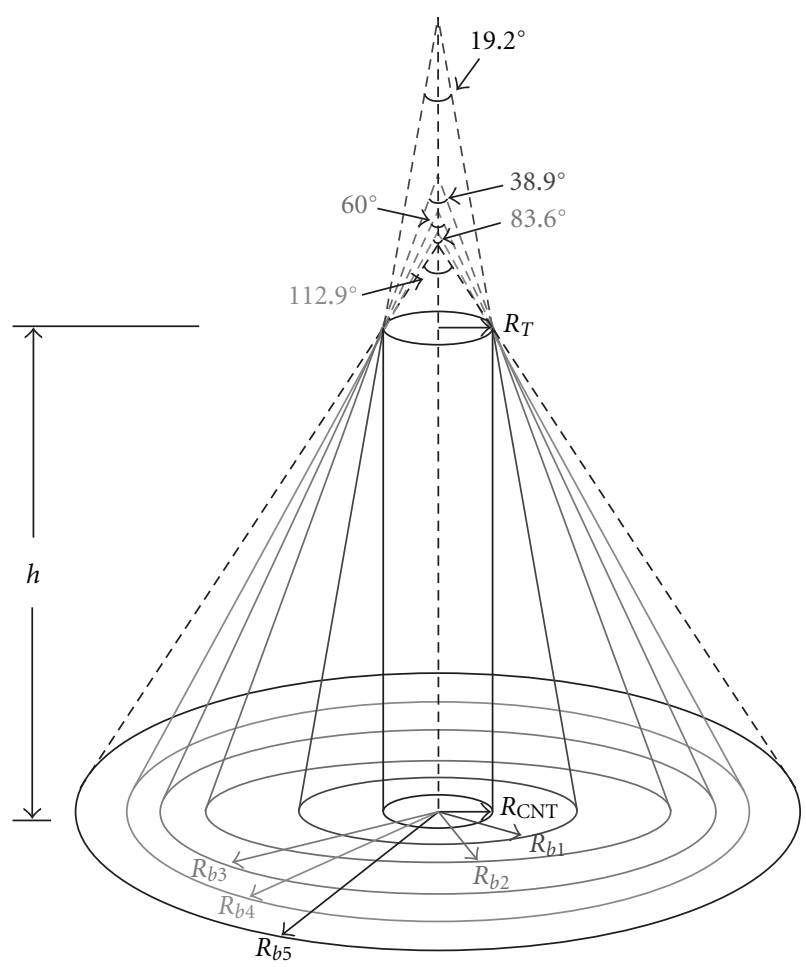

Figure 1: Open CNCs and a $(10,10)$ CNT with the same heights but different apex angles. $R_{T}=R_{\mathrm{CNT}}=6.8 \AA, R_{b 1}=17.1 \AA$, $R_{b 2}=28.4 \AA, R_{b 3}=39.7 \AA, R_{b 4}=51 \AA, R_{b 5}=62.3 \AA$, and $H=61.0 \AA$.

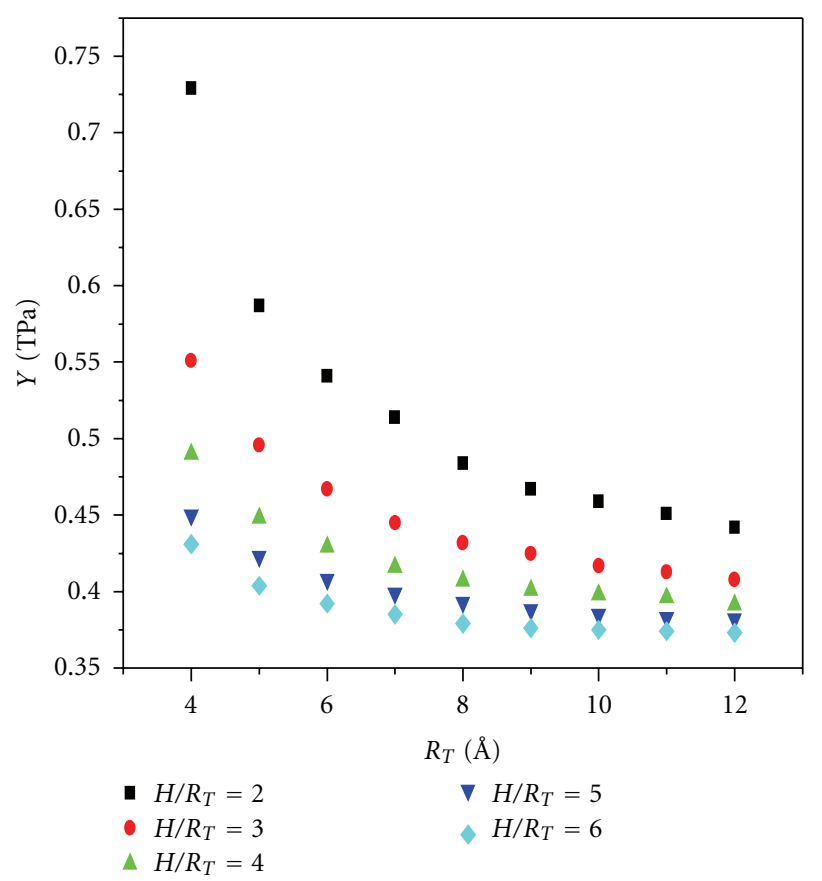

FIGURE 2: Young's modulus versus top radius with apex angle of $19.2^{\circ}$.

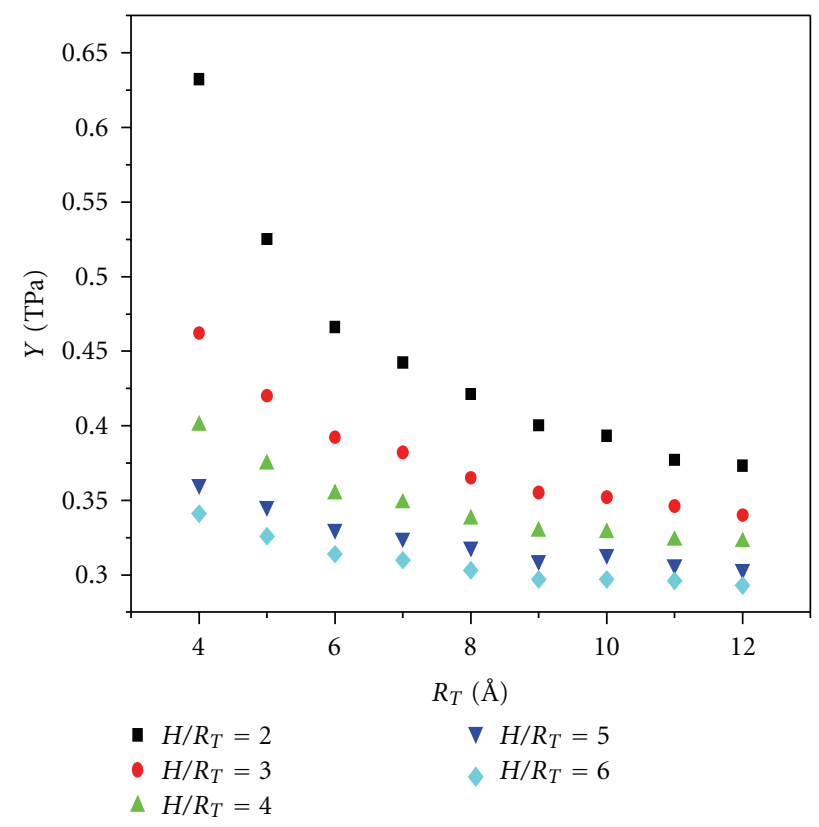

FIGURE 3: Young's modulus versus top radius with apex angle of $38.9^{\circ}$.

therefore the conventional stress-strain curves are not applicable. Simulation in CNHs is carried out by applying axial tensions at the top (smallest radius) end of the open $\mathrm{CNH}$, while at the same time keeping the other end fixed. Throughout the whole process, neither end of $\mathrm{CNH}$ is subjected to interatomic forces. As a result, top atoms move outwards along the axis and the inner atoms are relaxed while the end atoms are fixed. The whole $\mathrm{CNH}$ structure is therefore stretched in small steps. Young's Modulus of single shell carbon nanohorns varies directly proportional to $\cos ^{4} \theta$ of an equivalent single-wall carbon nanotube.

\section{Young's Modulus}

The Young's modulus $(Y)$ of the cone-shaped nanostructure can be determined by the second derivative of strain energy w.r.t. axial strain

$$
Y_{\mathrm{axis}}=\frac{d^{2} E}{V d \varepsilon^{2}}
$$

where $V$ is the overall volume of the cone shell

$$
V=\frac{t \pi}{\sin (\theta / 2)}\left(R_{b}^{2}-R_{a}^{2}\right)
$$

The thickness of wall is taken as $t=3.4 \AA$. Figures $2-4$ depict the variation of Young's modulus with top radius for three types of cones with apex angle of $19.2^{\circ}, 38.9^{\circ}$, and $60^{\circ}$. The young's modulus, which is often used to measure the strength of materials, is found to decrease with the increase in apex angle. 


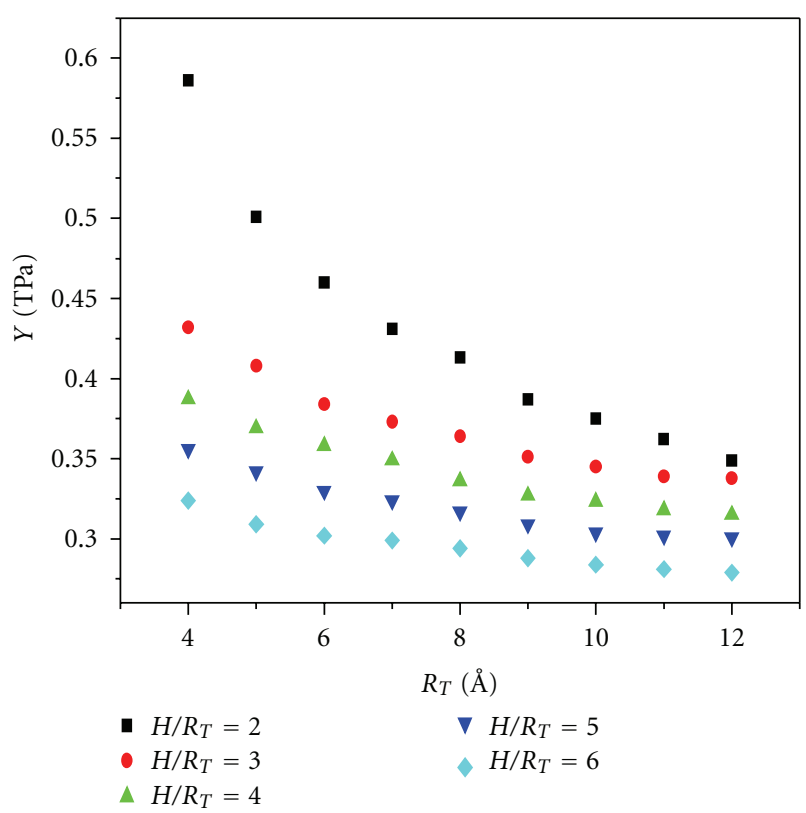

FIGURE 4: Young's modulus versus top radius with apex angle of $60^{\circ}$.

\section{Poisson Ratio}

For a single-shell nanohorn under a stress along the cone axis, coordinates are defined such that $X$ and $Y$ are along the radial directions and $Z$ is along the cone axis, with the corresponding strains as $\varepsilon_{x}, \varepsilon_{y}$, and $\varepsilon_{z}$. As $\mathrm{C}-\mathrm{C}$ bonds on the tilted graphene plane take the actual load, the axial strain, the length of the cone, and its change along the graphene plane are introduced as $\varepsilon_{z, \|}, L_{\|}$, and $\delta L_{\|}$, respectively, as shown in Figure 5.

Geometric considerations show that $L_{\|}+\delta L_{\|}=$ $\sqrt{(L \tan \theta)^{2}+(L+\delta L)^{2}}$, where $\gamma=-\varepsilon_{x, y} / \varepsilon_{z}$ is the Poisson ratio and $\delta L$ is the change of length $L$ along the $Z$ axis. The relation between $\varepsilon$ and $\varepsilon_{\| \mid}$thus can be expressed as

$$
\varepsilon_{z, \|}=\frac{\delta L_{\|}}{L_{\|}}=\left(\cos ^{2} \theta-\gamma \sin ^{2} \theta\right) \varepsilon_{z}+O\left(\varepsilon_{z}^{2}\right) .
$$

Omitting the higher-order terms in $\varepsilon$, a corresponding relation for the Poisson ratio along the graphene plane can be expressed as $\gamma_{\|}=-\varepsilon_{r} / \varepsilon_{z, \|}=-\varepsilon_{x, y} / \varepsilon_{z, \|}=\gamma /\left(\cos ^{2} \theta-\gamma \sin ^{2} \theta\right)$, where $\varepsilon_{r}$ is the strain in the radial direction. By neglecting the higher-order terms in $\gamma$, the Poisson ratio is further written as

$$
\gamma=\gamma_{\|} \cos ^{2} \theta
$$

As the strain energy $E=(1 / 2) k \varepsilon^{2}$ can be equivalently expressed as a function of $\varepsilon_{z}$ or $\varepsilon_{z, \|}$, the corresponding relation for the force constant $k$ and its counterpart $k_{\|}$in the graphene plane can be written as $k=k_{\|} \cos ^{4} \theta\left(1-\gamma_{\|} \sin ^{2} \theta\right)^{2}$. The Young's modulus of a single-shell nanocone is thus expressed as,

$$
Y_{\text {cone }}=Y_{\text {SWNT }} \cos ^{4} \theta\left(1-\gamma_{\|} \sin ^{2} \theta\right)^{2},
$$

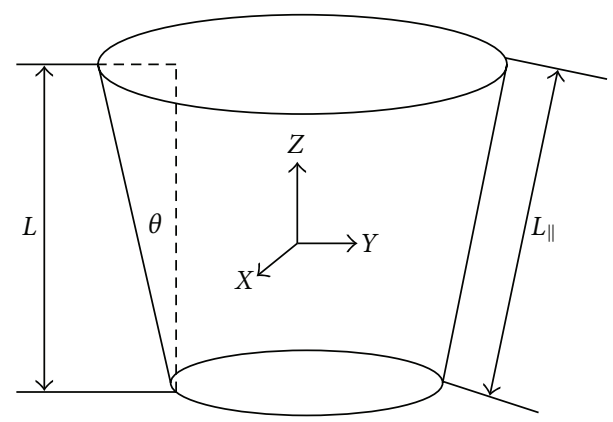

FIgURE 5: Illustration of a single-shell nanocone.

where $Y_{\text {SWNT }}$ is Young's modulus of CNTs and the wall thickness of the cone and the CNT is assumed the same as $h_{0}$.

For a wide-angle nanocone of $\theta=30^{\circ}, R_{1} \sim 15 \AA$, $R_{2} \sim 3 \AA$, and $L \sim 20 \AA$, axial strains of up to $5 \%$ were applied. Neglecting the radii change due to the Poisson ratio effect, the force constant for the nanocone is found to be $25.70 \mathrm{eV} /$ atom. Similarly, using REBO, it was found to be $45.70 \mathrm{eV} /$ atom on straight CNTs, and the value does not depend strongly on the radius and chirailty for $r>$ 3.5 A. Using these force constants in the relation $Y_{\text {cone }} \approx$ $Y_{\text {SWNT }} \cos ^{4} \theta$ (with $\gamma=0$ ) in (8), a value of $\theta=30.01^{\circ}$ is obtained, which is in excellent agreement with $\theta=$ $30^{\circ}$ from the geometry shown in Figure 5. Similarly, the MD-simulated $\gamma \approx 0.25$ for the nanocone is in excellent agreement with $\gamma \approx 0.23$ as predicted by (6) for $\gamma_{\|} \approx 0.30$ simulated for large diameter CNTs. Thus, our results validate (7) and [8] for the single-shell nanohorns.

\section{Shear Modulus}

A lateral force, in the form of a twist, is applied to the cone of a given top radius and height, keeping one of its ends fixed. The total torsion that has to be given to the cone has been distributed equally along the whole length of the cone and again coordinates of the cone in minimum energy configuration are obtained.

Based on the theory of elasticity, shear modulus at the macroscopic scale is given by

$$
G=\frac{T l_{o}}{\theta J(t)}
$$

where $T, l_{o}, \theta$, and $J(t)$ stand for the torque acting at the end of the $\mathrm{CNH}$, the length of the cone, the total torsion angle that is applied to the cone, and the cross-sectional polar moment of inertia of the nanocone, respectively,

$$
J(t)=\frac{\pi}{2}\left[\left(R_{a}+\frac{t}{2}\right)^{4}-\left(R_{a}-\frac{t}{2}\right)^{4}\right] .
$$

The shear modulus for three different cones is shown in Figures 6-8. The trends are the same as for Young's modulus. 


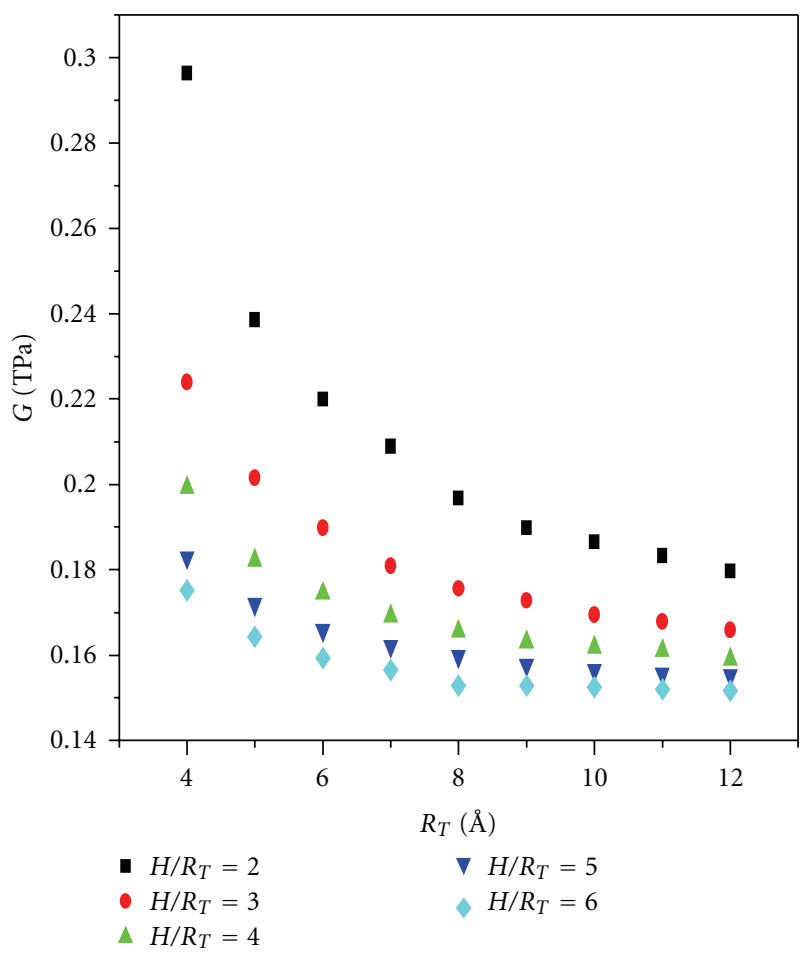

Figure 6: Shear modulus versus top radius with apex angle of $19.2^{\circ}$.

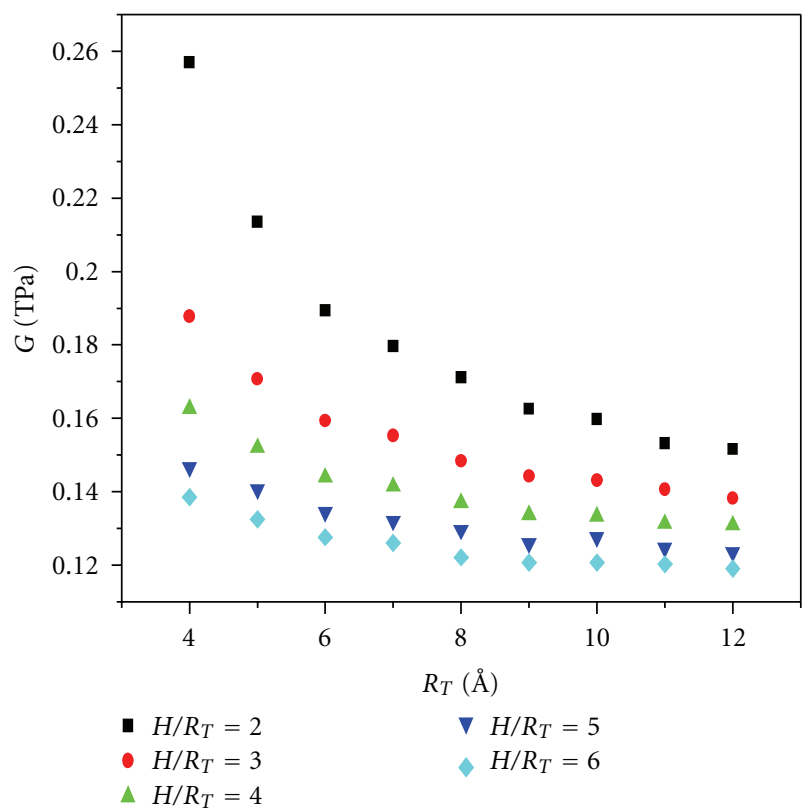

FIGURE 7: Shear modulus versus top radius with apex angle of $38.9^{\circ}$.

\section{Discussion and Conclusion}

For the carbon nano cones, the value of Young's modulus lies in the range of $0.24 \mathrm{Tpa}$ to $0.73 \mathrm{Tpa}$, and the Shear modulus varies from $0.10 \mathrm{Tpa}$ to $0.29 \mathrm{Tpa}$ as the top radius is decreased. Both moduli decrease with the increase in

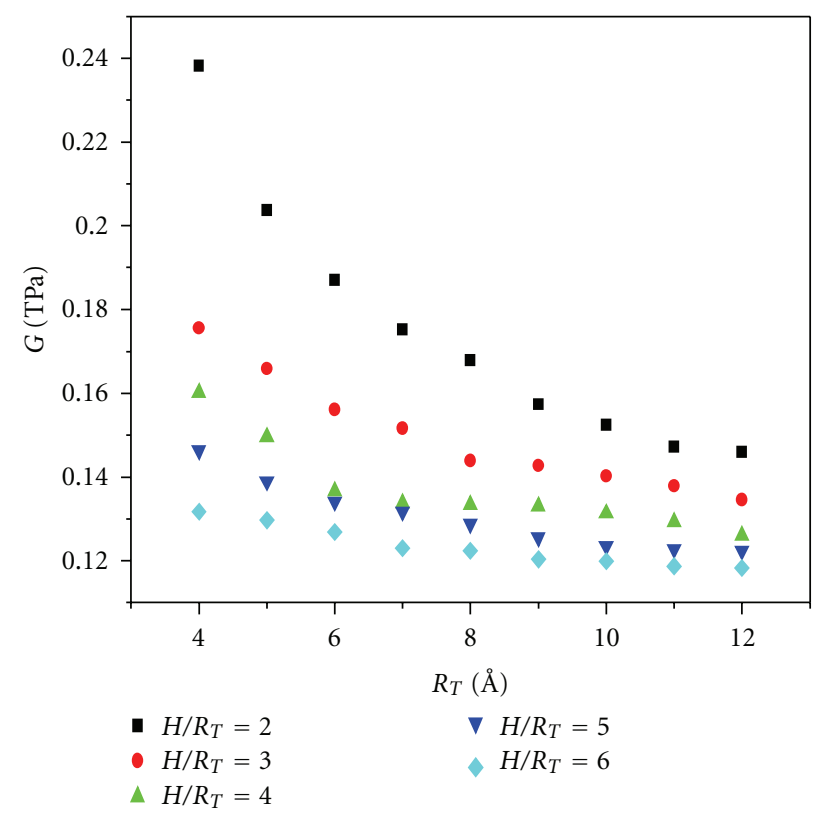

FIGURE 8: Shear modulus versus top radius with apex angle of $60^{\circ}$.

the radius or height of CNCs. For a fixed height to top radius ratio, the moduli are found to reach their limit once the top radius approaches certain level. For CNTs, the value of Young's modulus is around 1TPa, and the Shear modulus is of the order of $300 \mathrm{GPa}$ [22]. The value of Poisson's ratio for nanohorns is 0.23 , and for CNTs, the value is around 0.15 [22]. We have tried to understand these structures using model potential by Brenner and coworkers and conclude that nanohorns are equally strong in comparison to carbon nanotubes. These must be explored experimentally as well as theoretically for their potential applications.

\section{References}

[1] S. Iijima, "Helical microtubules of graphitic carbon," Nature, vol. 354 , no. 6348 , pp. 56-58, 1991.

[2] D. Ugarte, "Curling and closure of graphitic networks under electron-beam irradiation," Nature, vol. 359, no. 6397, pp. 707-709, 1992.

[3] Y. Saito and T. Matsumoto, "Carbon nano-cages created as cubes," Nature 392, pp. 237-244, 1998.

[4] Y. Saito, K. Nishikubo, K. Kawabata, and T. Matsumoto, "Carbon nanocapsules and single-layered nanotubes produced with platinum-group metals ( $\mathrm{Ru}, \mathrm{Rh}, \mathrm{Pd}, \mathrm{Os}, \mathrm{Ir}, \mathrm{Pt})$ by arc discharge," Journal of Applied Physics, vol. 80, no. 5, pp. 30623067, 1996.

[5] M. Ge and K. Sattler, "Observation of fullerene cones," Chemical Physics Letters, vol. 220, no. 3-5, pp. 192-196, 1994.

[6] A. Krishnan, E. Dujardin, M. M. J. Treacy et al., "Graphitic cones and the nucleation of curved carbon surfaces," Nature, vol. 388, no. 6641, pp. 451-454, 1997.

[7] D. H. Robertson, D. W. Brenner, and J. W. Mintmire, "Energetics of nanoscale graphitic tubules," Physical Review B, vol. 45, no. 21, pp. 12592-12595, 1992. 
[8] G. Oerney, W. Zhong, and D. Tománek, "Structural rigidity and low frequency vibrational modes of long carbon tubules," Zeitschrift für Physik D Atoms, Molecules and Clusters, vol. 27, no. 1, pp. 93-96, 1993.

[9] C. F. Cornwell and L. T. Wille, "Elastic properties of singlewalled carbon nanotubes in compression," Solid State Communications, vol. 101, no. 8, pp. 555-558, 1997.

[10] N. Yao and V. Lordi, "Young's modulus of single-walled carbon nanotubes," Journal of Applied Physics, vol. 84, no. 4, pp. 1939$1943,1998$.

[11] T. Belytschko, S. P. Xiao, G. C. Schatz, and R. S. Ruoff, "Atomistic simulations of nanotube fracture," Physical Review $B$, vol. 65, no. 23, Article ID 235430, pp. 1-8, 2002.

[12] E. Hernández, C. Goze, P. Bernier, and A. Rubio, "Elastic properties of single-wall nanotubes," Applied Physics A: Materials Science and Processing, vol. 68, no. 3, pp. 287-292, 1999.

[13] G. Dereli and C. Ozdogan, "Structural stability and energetics of single-walled carbon nanotubes under uniaxial strain," Physical Review B, vol. 67, no. 3, Article ID 035416, pp. 1-6, 2003.

[14] Z. Xin, Z. Jianjun, and O. U. Y. Zhong-can, "Strain energy and Young's modulus of single-wall carbon nanotubes calculated from electronic energy-band theory," Physical Review B, vol. 62, no. 20, pp. 13692-13696, 2000.

[15] D. Sánchez-Portal, E. Artacho, J. M. Soler, A. Rubio, and P. Ordejón, "Ab initio structural, elastic, and vibrational properties of carbon nanotubes," Physical Review B, vol. 59, no. 19, pp. 12678-12688, 1999.

[16] G. Van Lier, C. Van Alsenoy, V. Van Doren, and P. Geerlings, "Ab initio study of the elastic properties of single-walled carbon nanotubes and graphene," Chemical Physics Letters, vol. 326, no. 1-2, pp. 181-185, 2000.

[17] S. Ogata and Y. Shibutani, "Ideal tensile strength and band gap of single-walled carbon nanotubes," Physical Review B, vol. 68, no. 16, Article ID 165409, pp. 1-4, 2003.

[18] J. Wu, K. C. Hwang, and Y. Huang, "An atomistic-based finitedeformation shell theory for single-wall carbon nanotubes," Journal of the Mechanics and Physics of Solids, vol. 56, no. 1, pp. 279-292, 2008.

[19] J. Wu, K. C. Hwang, and Y. Huang, "A Shell Theory for Carbon Nanotubes Based on the Interatomic Potential and Atomic Structure," Advances in Applied Mechanics, vol. 43, pp. 1-68, 2009.

[20] D. W. Brenner, O. A. Shenderova, J. A. Harrison, S. J. Stuart, B. Ni, and S. B. Sinnott, "A second-generation reactive empirical bond order (REBO) potential energy expressions for hydrocarbons," Journal of Physics Condensed Matter, vol. 14, no. 4 , p. 783, 2002.

[21] D. Kumar and V. Verma, "Second generation (REBO) potential parameters for Carbon Nanotubes," International Journal of Nanosystems, vol. 1, pp. 49-53, 2008.

[22] S. Gupta, K. Dharamvir, and V. K. Jindal, "Elastic moduli of single-walled carbon nanotubes and their ropes," Physical Review B, vol. 72, no. 16, pp. 165428-165443, 2005. 

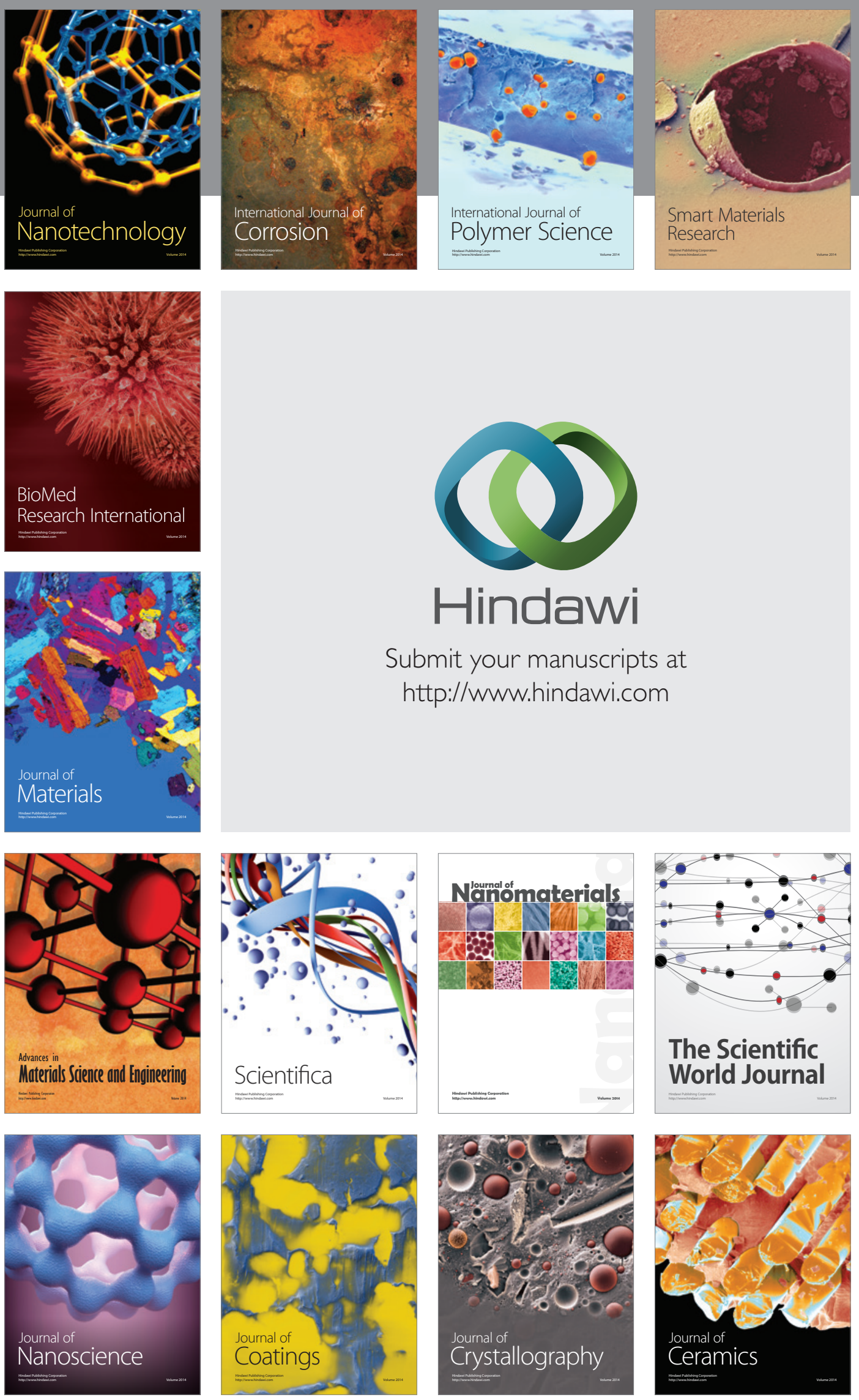

The Scientific World Journal

Submit your manuscripts at

http://www.hindawi.com

\section{World Journal}

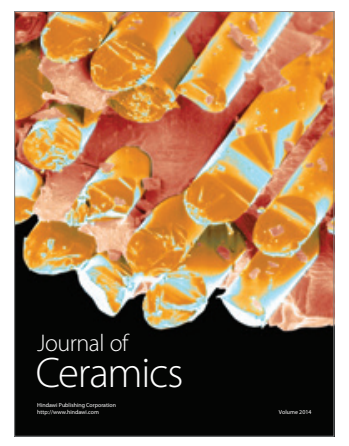

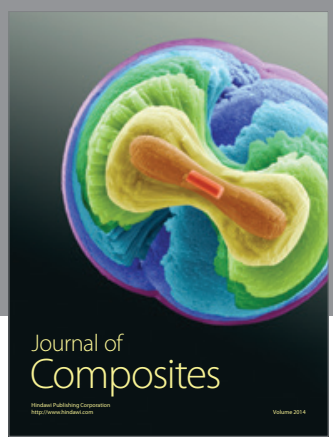
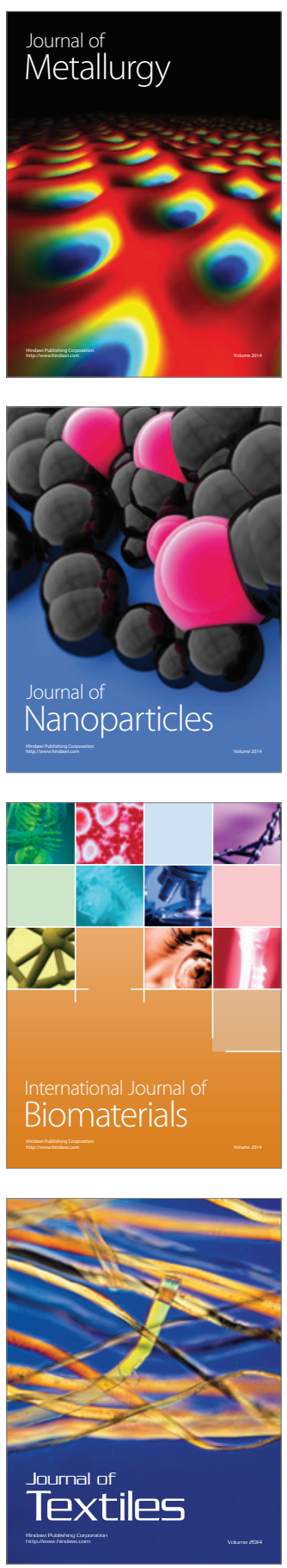